\title{
VIGILANCIA, PREVENCIÓN Y CONTROL DEL VIRUS DE LA INFLUENZA EN PERÚ
}

\author{
Victor Alberto Laguna-Torres ${ }^{1, a}$, Jorge Gómez $z^{2, b}$, Herminio Hernández ${ }^{3, \mathrm{c}}$, Jose Francia-Romero ${ }^{4, \mathrm{~d}}$, Aland Bisso-Andrade ${ }^{5, \mathrm{e}}$, \\ Alfredo Guerreros ${ }^{6, f}$, Jorge Cerna-Barco ${ }^{7, g}$, Eduardo Sanchez-Vergaray ${ }^{8,10, h}$, Eduardo Gotuzzo ${ }^{9, h}$
}

\begin{abstract}
RESUMEN
La importancia de la vigilancia de la circulación del virus de influenza y la vacunación oportuna a las diferentes poblaciones del Perú, fue analizada en tres sesiones por un grupo de clínicos expertos de diferentes especialidades. En Perú, en los últimos años, el sistema nacional de vigilancia de influenza tiene muchos establecimientos seleccionados que son ineficaces en la notificación oportuna. La mayoría de las muestras provienen de Lima. La distribución viral es inadecuadamente analizada. El norte y la selva tienen estaciones climáticas diferentes a Lima y la sierra sur. Esta descoordinación se acentúa con la oportunidad de la llegada de la vacuna de influenza al país. El Ministerio de Salud utiliza una vacuna trivalente pero la posibilidad de una vacuna tetravalente con los dos linajes de tipo B, debe ser analizada desde el punto de vista de costo-beneficio. Se debería evaluar la pertinencia de vacunar en dos momentos anuales, regionalmente.
\end{abstract}

Palabras clave: Vigilancia sanitaria; Prevención \& control; Gripe Humana; Vacunas contra la Influenza (Fuente: DeCS BIREME).

\section{SURVEILLANCE, PREVENTION, AND CONTROL OF INFLUENZA VIRUS IN PERU}

\begin{abstract}
The importance of surveilling the circulation of the influenza virus and timely vaccination of different populations in Peru was analyzed in three sessions by a group of clinical experts from different specialties. The Peruvian national influenza surveillance system has many selected establishments that have become currently ineffective in terms of a timely report. Most of the samples come from Lima. The viral distribution is inadequately analyzed. The northern and jungle areas of the country have weather and season conditions different to those in Lima and on the southern highlands. This lack of coordination is accentuated by the opportunity of arrival of the influenza vaccine in the country. The Ministry of Health uses a trivalent vaccine but the possibility of a tetravalent vaccine with the two type-B lineages must be analyzed from a cost-benefit standpoint. The appropriateness of vaccinating two times a year, regionally, should be assessed.
\end{abstract}

Keywords: Health Surveillance; prevention \& control; Influenza, Human; Influenza Vaccines (source: MeSH NLM).

\section{INTRODUCCIÓN}

El virus de la influenza es de transmisión respiratoria y tiene como característica principal sufrir anualmente mutaciones puntuales. Tiene, además, unagran capacidad de transmisión entre contactos cercanos, ocasionando que grandes grupos de personas, en una determinada época del año, presenten el llamado síndrome gripal que se produce cuando el virus ha vencido las defensas respiratorias de nuestro organismo ocasionando tos, fiebre elevada y malestar general ${ }^{(1)}$. Algunas personas infectadas se complican y evolucionan a neumonía por lo que tienen que hospitalizarse ocasionando eventos que podrían terminar en unidades de cuidados intensivos con alto riesgo de muerte (2).

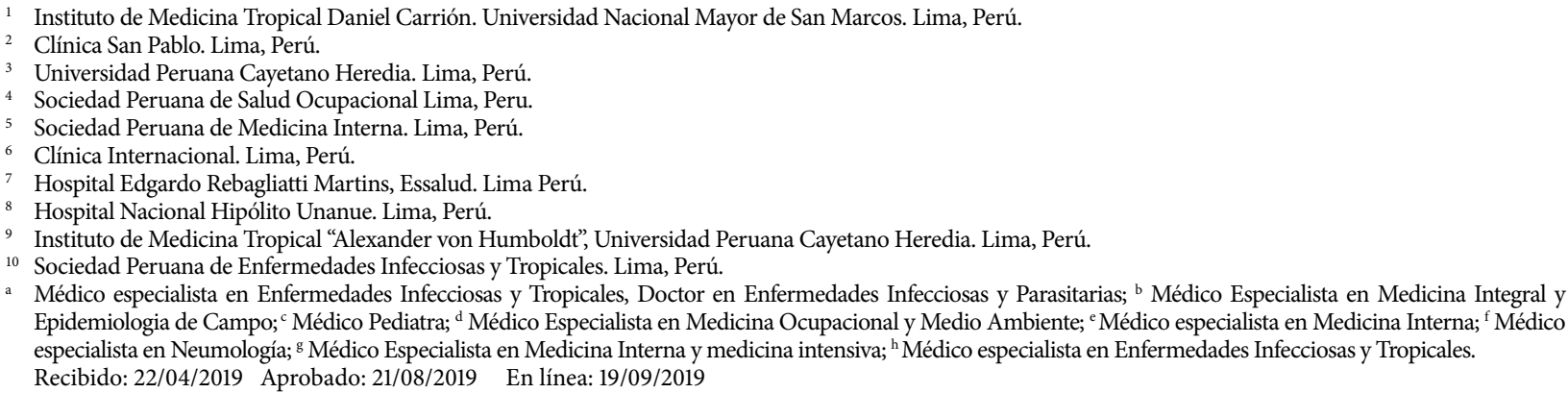

Citar como: Laguna-Torres VA, Gómez J, Hernández H, Francia-Romero J, Bisso-Andrade A, Guerreros A, et al. Vigilancia, prevención y control del virus de la influenza en Perú. Rev Peru Med Exp Salud Publica. 2019;36(3):511-4. doi: http://dx.doi.org/10.17843/rpmesp.2019.363.4481. 
El virus de la influenza es inestable y altamente impredecible, aunque usualmente se autolimita, ocasionalmente puede desarrollar complicaciones cardiacas, neurológicas y respiratorias ${ }^{(3,4)}$. Un patrón peculiar del virus es que tiene una interacción con el Streptococcus pneumoniae que ocasiona infecciones subsecuentes que comprometen al parénquima pulmonar ${ }^{(5-7)}$

Cada año, los casos de Influenza aumentan a nivel mundial y de manera similar aumenta la mortalidad, hace ocho años se calculaba entre 250000 y 400000 el número de fallecidos por influenza; sin embargo, en los últimos dos años se considera que hay más de 650000 muertes; de manera importante en los grupos de riesgo ${ }^{(8)}$.

En el 2018, la onda de influenza se ha notado más agresiva en Norteamérica, en países de Centro América y países que ya pasaron el invierno, como Colombia y Ecuador, con mayores hospitalizaciones, uso de respiradores, complicaciones neumónicas postinfluenza y muerte.

Establecer un sistema de vigilancia que muestre la distribución de la circulación viral en los diferentes estratos geográficos del Perú es vital. En ese sistema, la vigilancia de tipo centinela debe ser capaz de alertar sobre la presencia de subtipos circulantes y aún más estar asociada a un laboratorio de referencia con capacidad diagnóstica y de establecer la tipificación molecular de los virus circulantes y si ellos se asociación con la cepa vacunal que se aplica a la población peruana. Asimismo, el sistema debe vigilar constante y eficazmente que tipo de influenza circula en los hospitalizados y en las unidades de cuidados intensivos del país.

Es así que, el segundo semestre del 2018, clínicos expertos de diferentes especialidades e instituciones participamos de tres sesiones privadas con el fin de analizar la situación de la vigilancia, prevención y control de la infección por el virus de la influenza en el Perú. La búsqueda de consensos que puedan ser útiles para las autoridades sanitarias del país fue el objetivo principal del grupo.

\section{VIGILANCIA DE LA INFLUENZA EN EL PERÚ}

El Perú estableció un sistema de vigilancia centinela y de infecciones respiratorias agudas graves (IRAG) muy eficaz ${ }^{(9,10)}$ y que durante la pandemia del 2009 analizó y caracterizó la situación epidemiológica y brindó respuestas oportunas para la salud pública del país ${ }^{(11-13)}$. El sistema había establecido que en la costa norte (Piura) el virus de la influenza circulaba todo el año ${ }^{(9)}$ siendo los casos notificados con frecuencia en enero y febrero, antes de la temporada de invierno de Lima y de la sierra. En la región amazónica, Loreto tendría un comportamiento similar al del norte del país con algunas peculiaridades. Por otro lado, en Cusco, Arequipa y Puno (9) la circulación viral se identificó principalmente en la época de friaje de la zona altoandina (abril-agosto). La naturaleza cambiante del virus y las características de su circulación hace necesaria que la vigilancia sea constante y muestre las variaciones anuales que puedan presentarse ${ }^{(13)}$. La vigilancia centinela necesita que establecimientos seleccionados de un área específica, capten una cuota de pacientes con síndrome gripal y que su muestra sea examinada por el método qPCR. En una región no se necesita más de un establecimiento centinela pero que sea eficiente.

De acuerdo al reporte de muestras que se notifican de las sedes de vigilancia, estimamos que, en los últimos años, el sistema tiene muchos establecimientos seleccionados y muchos de ellos notifican una o ninguna muestra en un año, al final la mayoría de las muestras analizadas en la vigilancia provienen de establecimientos de Lima y de la temporada en que Lima se ve afectada por el frío. Eso ocasiona que la distribución viral sea inadecuadamente analizada $^{(14,15)}$. El norte y la selva peruana tienen estaciones climáticas diferentes a Lima y la sierra sur peruana. Esta descoordinación se acentúa con la oportunidad de la llegada de la vacuna de influenza al país. En el Perú se usa la vacuna formulada para el hemisferio sur, la que usualmente está accesible al final de abril o comienzos del mes de mayo y su distribución al país se da usualmente cuando los casos de síndrome gripal por influenza ya están diseminados por Lima y las zonas alto andinas del sur del país; sin embargo, con la población de Piura y norteños en general, el tema es más álgido, puesto que reciben la vacuna en junio o julio cuando muchos de ellos se enfermaron en el primer bimestre del año.

Es importante señalar que, debido al comportamiento del virus en Perú, se debería discutir la pertinencia de vacunar en dos momentos por año. Los pobladores del norte y los de la selva podrían recibir la vacuna en enero o febrero a diferencia de Lima y la sierra sur. Se sabe que difícilmente Perú contará con la vacuna del hemisferio sur en el mes de enero, pero podría ser que las cepas circulantes en esa área del país, estén incluidas en la vacuna del hemisferio norte, la cual, sí está accesible en diciembre para muchos países. La vigilancia epidemiológica eficiente debe, anualmente, brindarnos esa información y permitir que se pueda determinar lo adecuado.

\section{PREVENCIÓN Y CONTROL}

La vacunación es la medida más efectiva de prevención ${ }^{(16,17)}$. Esta vacunación anual debe ser administrada en los grupos de alto riesgo de infectarse y llegar a complicaciones como hospitalizarse y/o fallecer. Estos grupos son los niños menores de cinco años (entre seis meses - cinco años), los adultos mayores de 65 años, las gestantes de cualquier semana de gestación; las personas con enfermedades crónicas como diabetes, insuficiencia renal, enfermedad pulmonar crónica, obesidad, enfermedad cardiovascular (17). Asimismo, en 
pacientes con inmunodeficiencias por quimioterapia, VIH/ Sida, síndrome de Down, entre otros.

Recientemente se ha considerado controversial el uso del oseltamivir, antiviral que saltó a la fama durante la pandemia del 2009. Una revisión sistemática en el 2003 mostró que el uso del fármaco reducía complicaciones y riesgo de hospitalización ${ }^{(18)}$, pero en la revisión sistemática del 2014 realizada por el Cochrane Acute Respiratory Infections Group ${ }^{(19)}$ se mostró que el beneficio de su uso es reducido. Una revisión hecha por el Instituto Nacional de Salud del Perú, afirma que el oseltamivir consigue reducción modesta de casi un día de la duración de los síntomas de influenza en adultos y niños, y no produce ningún efecto significativo en ancianos o pacientes en riesgo ${ }^{(20)}$. La falta de un tratamiento antiviral para poder combatir adecuadamente la infección por influenza hace que se enfatice en la vacunación, la cual es prácticamente el único medio farmacológico eficaz que tenemos para prevenir la infección viral.

La vacuna contra la influenza se formula incluyendo las cepas que la vigilancia hemisférica ha mostrado como predominante ${ }^{(17)}$. Incluye cepas del subtipo H1N1, H3N2 y $\mathrm{B}$. Puede entonces ser trivalente o también tetravalente, cuando se incluyen dos subtipos B para dar cobertura a todas las posibilidades de infección contra el subtipo B. La utilización del tipo tetravalente daría una ventaja disminuyendo la morbilidad por el tipo B. La vacuna es eficaz desde los seis meses de edad y debe aplicarse en mayores de 65 años y menores de cinco años. Asimismo, al personal de salud, gestantes, personas con enfermedades crónicas, hipertensas, diabéticas y militares ${ }^{(21,22)}$. Las gestantes con Influenza tienen elevada tasa de hospitalización y complicaciones en comparación con mujeres no gestantes de la misma edad con influenza. La vacunación de la gestante protege, además, al recién nacido que no puede ser vacunado hasta cumplir seis meses. Usualmente, nuestras poblaciones altoandinas terminan vacunadas cuando ya el frío y las complicaciones clínicas suelen estar presentes.

Los programas de vacunación dentro del Perú ocupan una actividad importante, pero las coberturas aún están debajo de lo esperado ${ }^{(23)}$. Es básico transmitir el concepto que las vacunaciones son una de las dos medidas más importantes que permiten reducir morbilidad, hospitalización y muerte en nuestra población.

Es importante resaltar que, el personal de salud presenta mayor riesgo porque está en contacto con múltiples secreciones de pacientes potencialmente infectados. Sin embargo, este grupo profesional suele rechazar frecuentemente la aplicación. El trabajo motivacional será de gran utilidad. La vacunación debe independizarse de temas administrativos o ministeriales y debe ser una política de estado.

La utilidad de la vacuna supera por mucho los ocasionales efectos adversos. La Organización Mundial de la Salud (OMS) por años ha recomendado la vacuna trivalente que incluye los tres virus más notables cada año (vacuna trivalente que se usa en la actualidad en el Perú).

Es importante mencionar que las cepas del tipo B son de dos linajes: Yamagata y Victoria, los cuales circulan sin mayor periodicidad y la inclusión de ambos linajes en la vacuna conforma la vacuna tetravalente (dos virus tipo A-H1N1 y H3N2, así como dos linajes del tipo B Yamagata y Victoria). La eficiencia de la vacuna tetravalente es elevada y reduce las formas graves y las neumonías bacterianas como complicación temprana de infecciones. La seguridad es adecuada tanto para niños como para adultos y para personas de la tercera edad. Ambas vacunas tri y tetravalente están aprobadas en el Perú. La tetravalente es ampliamente usada en el sector privado con la ventaja que aumenta la cobertura de cepas. La necesidad actual de usar la vacuna tetravalente se hace cada vez más importante y necesaria. Deberá ser evaluada por los expertos del Ministerio de Salud, así como las ventajas económicas de su incorporación en las estrategias anuales de vacunación nacional.

\section{CONCLUSIONES}

Es indispensable hacer vigilancia epidemiológica y molecular para saber oportunamente qué tipo de virus Influenza nos está afectando. Investigaciones previas señalan que tenemos dos patrones diferentes: a) Circulación estacional en Lima y zona del Sur (Arequipa, Puno, Cusco) y b) Circulación casi continua en el norte y en la amazonia. La vigilancia permitirá hacer un plan de vacunación basada en datos peruanos. Asimismo, es necesario desmitificar la vacunación tanto en los profesionales médicos como en la población general, estableciendo claramente los criterios para vacunar, las posibles contraindicaciones y mejorar la comunicación médico-paciente.

Agradecimiento: a la Dras Grécia Egúsquiza y María Fernanda Farías, por su apoyo en la sistematización de los conceptos discutidos durante las tres reuniones.

Contribuciones de autoría: Los autores participaron en la concepción, diseño y aprobación final del manuscrito.

Conflictos de interés: Los autores declaran que no tienen conflicto de interés. Dr. Victor Laguna ha participado como ponente en temas relacionados a vacunas e infecciones respiratorias, convocado por diferentes laboratorios. Eduardo Gotuzzo ha participado en asesoría de Laboratorios Sanofi y MSD.

Fuentes de financiamiento: Este trabajo y las reuniones de los expertos fue efectuado con un grant recibido por la Universidad Peruana Cayetana Heredia. 


\section{REFERENCIAS BIBLIOGRÁFICAS}

1. Cross K, Burleigh L, and Steinhauer D. Mechanisms of cell entry by influenza virus. Expert Rev Mol Med. 2001;3(21): 1-18.

2. Schwarzmann $S$, Bacterial pneumonia during the Hong Kong influenza epidemic of 1968-1969. Arch Intern Med. 1971;127(6):1037-41.

3. Rezkalla $S$, and Kloner R. Influenzarelated viral myocarditis. WMJ. 2010;109(4):209-13.

4. Amin R. Ford-Jones E, Richardson SE, MacGregor D, Tellier R, Heurter $\mathrm{H}$, et al. Acute childhood encephalitis and encephalopathy associated with influenza: a prospective 11-year review. Pediatr Infect Dis J. 2008;27(5):390-5.

5. McCullers J. Insights into the interaction between influenzavirusand pneumococcus. Clin Microbiol Rev. 2006; 19(3): 571-82.

6. McCullers J, Bartmess K. Role of neuraminidase in lethal synergism between influenza virus and Streptococcus pneumoniae. J Infect Dis. 2003; 187(6): 1000-9.

7. Madhi S, Klugman K. A role for Streptococcus pneumoniae in virusassociated pneumonia. Nat Med. 2004;10(8): 811-3.

8. World Health Organization. Up to 65000 people die of respiratory diseases linked to seasonal flu each year [Internet]. Ginebra: who; 2018 [citado el 1 de mayo 2018]. Disponible en: www.who. $\mathrm{int} /$ news-room/14-12-2017-up-to-650000-people-die-of-respiratory-diseaseslinked-to-seasonal-flu-each-year

9. Laguna-Torres VA, Gomez J, Ocaña V, Aguilar P, Saldarriaga T, Chavez E, et al., Influenza-like illness sentinel surveillance in Peru. PLoS One. 2009;4(7): e6118.

10. Guía Técnica de Vigilancia de Influenza y otros virus respiratorios. Lima: Ministerio de Salud del Perú; 2007.
11. Munayco CV, Gomez J, Laguna-Torres VA, Arrasco J, Kochel TJ, Fiestas V, et $a l$, Epidemiological and transmissibility analysis of influenza $\mathrm{A}(\mathrm{H} 1 \mathrm{~N} 1) \mathrm{v}$ in a southern hemisphere setting: Peru. Euro Surveill, 2009; 14(32).

12. Gomez J, Munayco CV, Carrasco J. Suarez L, Laguna-Torres V, Aguilar P, et al. Pandemic influenza in a southern hemisphere setting: the experience in Peru from May to September, 2009. Euro Surveill, 2009; 14(42). pii: 19371. Doi: 10.2807/ese.14.42.19371-en

13. Laguna-Torres VA, Gomez J, Aguilar P. et al., Changes in the viral distribution pattern after the appearance of the novel influenza A H1N1 (pH1N1) virus in influenza-like illness patients in Peru. PLoS One, 2010; 5(7): el1719.

14. Medina, JL. Vigilancia de Influenza y otros virus respiratorios (OVR). Lima: Ministerio de Salud del Perú; 2018 [Citado el 1 de julio 2019]. Disponible en: https://www.dge.gob.pe/portal/ docs/tools/01.pdf

15. Reportes Anuales del Perú a OPS, FLUNET. [Citado el 1 de julio 2019]. Disponible en: http://ais.paho.org/ phip/viz/ed_flu.asp

16. Nicholson KG, Wood JM,ZambonM. Influenza. Lancet 2003; 362(9397):1733-4

17. Ropero-Alvarez AM, Whittembury A, Kurtis HJ, dos Santos T, DanovaroHolliday MC, Ruiz-Matus C. Pandemic influenza vaccination: Lessons learned from Latin America and the Caribbean. Vaccine.2012; 30 (2012): 916-921. doi: 10.1016/j.vaccine.2011.11.092.

18. Kaiser L, Wat C Mills T, Mahoney P, Ward P,Hayden F. Impact of oseltamivir treatment on influenza-related lower respiratory tract complications and hospitalizations. Arch Intern Med. 2003;163:1667-72.
19. Jefferson T, Jones M. Doshi P, Spencer EA, Onakpoya I, Heneghan CJ. Oseltamivir for influenza in adults and children: systematic review of clinical study reports and summary of regulatory comments. BMJ. 2014;348:g 2545. doi. org/10.1136/bmj.g2545

20. Aramburu A, Gutierrez E. Eficacia y seguridad de oseltamivir para el tratamiento de influenza. Lima: Instituto Nacional de Salud; 2017.

21. Saldarriaga T, Laguna-Torres VA, Arrasco J, Guillén L, Águila J, Aguilar P, et al, Características clínica y moleculares de un brote de influenza en dos bases militares, Tumbes- Perú, 2007. Rev Peru Med Exp Salud Publica. 2008; 25(1): 35-43.

22. Chavez E, Laguna-Torres VA, Burga AM, Sovero M, Cruz R, Pérez J, et al. Clinical and epidemiological characteristics of an influenza outbreak in military personnelTrujillo, Peru, 2008. Rev Peru Med Exp Salud Publica. 2010;27(3):373-8.

23. Equipo de Seguimiento Concertado a las Políticas de Salud Sub Grupo "Inmunizaciones en Niñas y Niños menores de 5 años". Reporte $\mathrm{N}^{\circ}$ 2-2017-SC/MCLCP Situación de la cobertura de vacunas para los menores de 3 años. Al primer semestre 2017. Lima: Mesa de concentración para la lucha contra la pobreza, 2017 [citado el 1 de diciembre 2018]. Disponible en https://www.mesadeconcertacion. org.pe/sites/default/files/ archivos/2017/documentos/09/ reportevacunasgtsalud $240817 \mathrm{vrev} 6$. pdf

\footnotetext{
Correspondencia: Victor Alberto Laguna

Torres

Correo electrónico:Laguna_alberto@hotmail.com
} 